\title{
Comparison of two different rest intervals on drop jump: effects on muscle damage markers
}

\author{
Alexandre E. Eiras, Rodrigo L. Reis, Pierre A. Silva, André N. Monteiro, Marco Machado \\ Laboratory of Physiology and Biokinetics - UNIG, Itaperuna, RJ, Brazil
}

\section{Summary}

Study aim: To determine the effects of 60- or 180-s rest intervals between sets of drop jump (DJ) exercise on serum creatine kinase (CK) activity and perceived muscle soreness.

Materials and methods: A group of 36 healthy male subjects aged 18 - 19 years performed 4 sets of $10 \mathrm{DJ}$, the rest intervals between sets amounting to 60 (Group A) or $180 \mathrm{~s}$ (Group B). Before, as well as 3, 24, 48, and 72 h postexercise, blood was sampled for determining the activity of in serum CK activity and the perceived muscle soreness was reported. Two-way ANOVA (time $\times$ rest interval) was used in data analysis.

Results: The DJ sessions induced muscle soreness and elevated serum CK activity $(\mathrm{p}<0.05)$. No significant betweengroup differences were found.

Conclusion: Rest intervals of $1-2$ min duration had no effect on the magnitude of changes in CK activity or perceived muscle soreness. This may be of practical importance when designing training which includes drop jumps.

\section{Key words Plyometric exercise - Muscle soreness - Muscle damage}

\section{Introduction}

Plyometric exercises were demonstrated to improve power output, agility and running economy. These exercises use rapid, powerful movements preceded by a preloading countermovement that activates the stretchshortening cycle (SSC). The SSC involves mechanical stretching of muscles and tendons immediately prior to rapid concentric contractions. The mechanisms thought to contribute to SSC include neural potentiation of the contractile machinery during the eccentric phase and reflect contributions from the muscle spindle [12]. One of the most commonly used plyometric exercises is the drop jump (DJ); this consists of stepping from a measured drop height and, on landing on the ground, to perform a maximal vertical jump with a short groundcontact period [14].

Drop jump is an eccentric exercise, the eccentric actions being associated with skeletal muscle tissue damage (sarcolemma disruption and sarcomere degeneration from Z-disk fragmentation). This induces a leak of skeletal muscle enzymes into blood serum, among them creatine kinase (CK), lactate dehydrogenase (LDH) and myoglobin, which were thus extensively used as markers for muscle micro-damages $[4,9,11,13]$. Under such conditions, CK concentrations in serum display greater increases than those of other muscle proteins. As a result, CK is used widely as such a marker of the status of muscle tissue $[1,4,8,9]$.

Plyometric exercises were investigated in many studies $[5,7,10,12]$ together with the effects of eccentric exercise on muscle damage markers [3,9,11], but the effects of SSC on those markers serum activity or of rest intervals between sets were rarely reported. As shown in other studies $[4,8,12]$, overtraining can be induced by high loads or high volumes of workout, which can induce micro-injuries and an immune-endocrine imbalance [6]. Thus, quantifying muscle damage markers in exercise protocols employing different rest intervals may help coaches and athletes to better prepare the training schedule. The aim of this study was thus to determine the effects of 60- or 180-s rest intervals between sets of drop jump (DJ) exercise on serum creatine kinase (CK) activity and perceived muscle soreness.

\section{Material and Methods}

Subjects: Thirty-six untrained subjects aged 18 to 19 years volunteered to participate in the study. They were healthy and did not use drugs or nutritional supplements. 
The subjects were randomly assigned into two groups, $\mathrm{n}$ = 18 each: A (60-s rest intervals) and B (180-s rest intervals). Subjects' characteristics are displayed in Table 1. Study objective and protocol were explained to the subjects who gave their informed consents according to the Declaration of Helsinki. The study was approved by the local Ethics Committee.

Table 1. Basic data of male subjects aged 18 - 19 years

\begin{tabular}{|c|c|c|}
\hline Group & $\begin{array}{c}\mathrm{A} \\
(\mathrm{n}=18)\end{array}$ & $\begin{array}{c}\mathrm{B} \\
(\mathrm{n}=18)\end{array}$ \\
\hline Age (years) & $18.6 \pm 0.5$ & $18.8 \pm 0.7$ \\
\hline Body mass (kg) & $66.5 \pm 12.1$ & $68.6 \pm 9.2$ \\
\hline Body height (cm) & $177.4 \pm 7.9$ & $178.0 \pm 6.5$ \\
\hline
\end{tabular}

Methodology: On the first day, the subjects underwent medical evaluation, body height and mass measurements and first blood sampling (PRE). Then they performed a 10-min warm-up (stretching and jogging) and performed 4 sets of maximal drop jumps (10 jumps per set) alternately on 2 one-m standard platforms $30 \mathrm{~cm}$ high. All the time the subjects were prompted vocally. The sets were separated by passive intermissions lasting 60 or $180 \mathrm{~s}$ in groups A and B, respectively. The time needed to execute individual DJ sets was measured.

After the exercise session was terminated, blood samples were collected 3, 24, 48 and $72 \mathrm{~h}$ after the first sampling. The subjects were requested not to engage in any kind of physical activity for the next 72 hours. The perceived muscle soreness was assessed at the same time points using a visual analogue scale $100 \mathrm{~mm}$ long, ranging from "not sore at all” (0) to "unbearably sore” (100 $\mathrm{mm})$ [2].

Venous blood samples were collected in seated position from the antecubital vein, the first sample (PRE) being collected in the morning, the other ones at the time points listed above. Blood was centrifuged and the separated serum was quickly frozen and stored at $-80^{\circ} \mathrm{C}$ until assayed for creatine kinase (CK) activity by an enzymatic method at $37^{\circ} \mathrm{C}$ using commercial kits (BioTécnica, Brazil) and Cobas Mira Plus analyser (Roche, Germany).

Data analysis: The data were subjected to two-way ANOVA (time $\times$ rest interval) followed by Tukey's post hoc test; the CK data were converted to logarithms prior to the analysis. Differences in CK values between 24 or $48 \mathrm{~h}$ post-exercise and the PRE state were calculated for computing Pearson's coefficients of correlation with muscle soreness. The level of $\mathrm{p} \leq 0.05$ was considered significant. SPSS ${ }^{\circledR} 15.0$ for Windows software was used.

\section{Results}

The applied plyometric exercise brought about significant $(\mathrm{p}<0.05-0.01)$ increases in the CK activity in serum (Fig. 1). However, no significant differences were found throughout $72 \mathrm{~h}$ post-exercise between the two groups of subjects. The same was true for muscle soreness (Fig. 2).

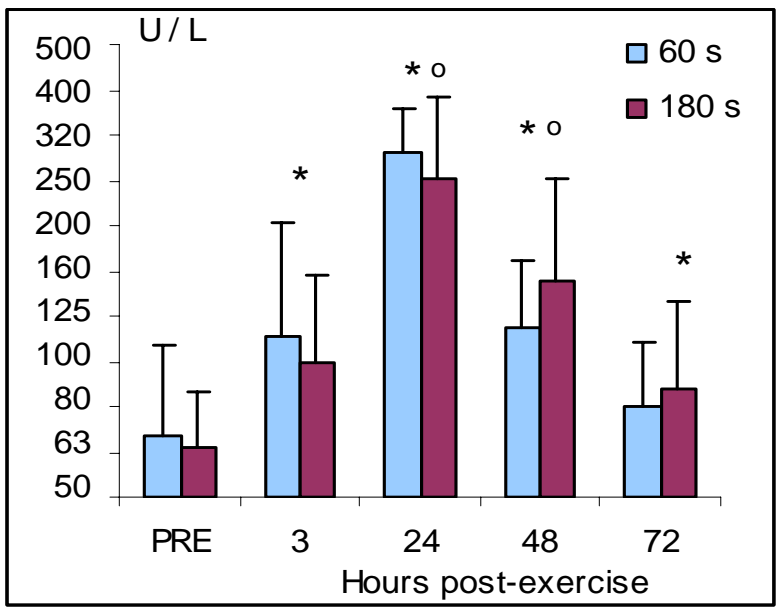

Fig. 1. Mean values $( \pm S D)$ of creatine kinase activity (log scale) at various times following a plyometric exercise Significantly $(\mathrm{p}<0.05)$ different: * From the PRE value; ${ }^{\circ}$ From the $3 \mathrm{~h}$ post-exercise value

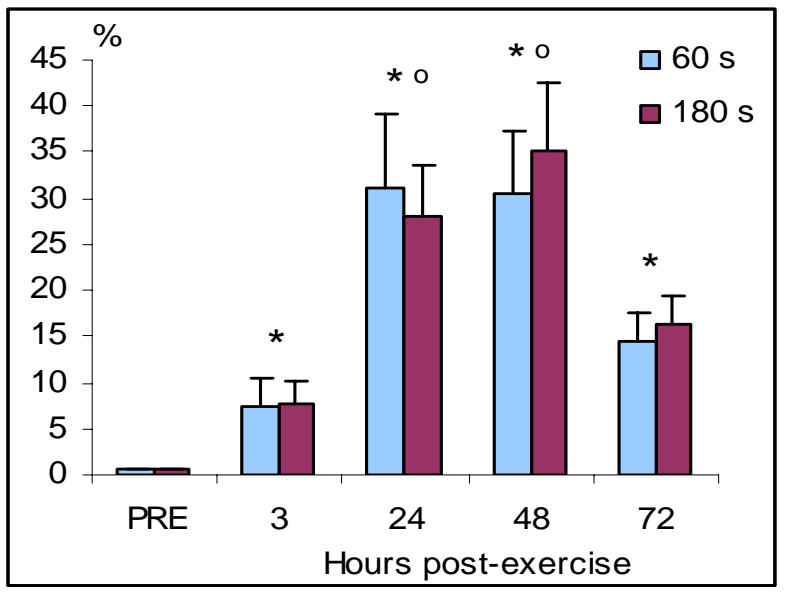

Fig. 2. Mean values $( \pm \mathrm{SD})$ of perceived muscle soreness at various times following a plyometric exercise

Significantly $(\mathrm{p}<0.05)$ different: * From the PRE value; ${ }^{\circ}$ From the $3 \mathrm{~h}$ post-exercise value

The 24-h increments in CK activities correlated significantly $(\mathrm{p}<0.05)$ with the perceived muscle soreness recorded $24 \mathrm{~h}$ post-exercise. No significant correlations were found for 48-h increments or for muscle soreness $48 \mathrm{~h}$ post-exercise (see Table 2). 
Table 2. Coeficients of correlation between perceived muscle soreness and changes in serum CK activity following a plyometric exercise $(n=36)$

\begin{tabular}{|c|c|c|}
\hline$\Delta \mathrm{CK}$ & $24 \mathrm{~h}$ & $48 \mathrm{~h}$ \\
\hline Muscle soreness & post-exercise & post-exercise \\
\hline $24 \mathrm{~h}$ post-exercise & $-0.332 *$ & -0.064 \\
\hline 48 h post-exercise & -0.227 & -0.047 \\
\hline
\end{tabular}

$* \mathrm{p}<0.05$

\section{Discussion}

Increases in the perceived post-exercise muscle soreness were consistent with those previously reported [3,9] and the same was true for serum CK activity [3,4,9]. These observations are indirectly indicative of muscle damage resulting from the plyometric exercise protocol. The serum CK activity serves as an index of both overexertion and adaptation of the muscular system to exercise. Total serum CK activity remained markedly elevated up to $48 \mathrm{~h}$ post-exercise and then gradually returned to basal levels (about $72 \mathrm{~h}$ post-exercise), the subjects resting. Some studies $[1,8]$ demonstrated that serum CK activity increased $3 \mathrm{~h}$ post-exercise and this effect was sustained up to $72 \mathrm{~h}$ post-exercise. This is consistent with our findings and may have been due to a large eccentric component of DJ. The present study corroborates previous reports where the $\mathrm{CK}$ activity remained elevated for 24 - 72 hours post-exercise [3, 8, 9,11].

A repeated stretching of the quadriceps during DJ might have led to disruptions of muscle fibres as a result of early fatigue and temporary increases in muscle stiffness due to the eccentric component [14]. Many authors $[3,4,9]$ reported that eccentric actions are the most potent in causing damages and increases in the activities of serum enzymes. As the eccentric actions predominate in DJ, high activities of serum CK and LDH were expected, especially due to the rapid eccentric muscle actions induced by the stretch reflex, which potentiate muscle effort and damage. In this study, CK activities increased about threefold, this being consistent with other studies, even using different methodologies. Mougios [8] proposed reference values for athletes of various sports, without specifying the type of training. Our data were within those proposed by Mougios [8]. It thus seems reasonable that the dosage of enzymes may be used to monitor plyometric training.

Muscle soreness, also following plyometric exercise, was studied by other authors; like Twist and Eston [14], we noted elevated muscle soreness induced by plyometric exercise. Similar data were reported also for other types of exercises [9,14]. Correlations between these variables were reported by others but in no study the methodology presented in this paper was used. This difference in methodology may have been responsible for the disparity in relation to other studies.Summing up, the here presented data are consistent with other reports that drop jumps induce increased serum CK activity, the rest intervals of $1-2$ min duration having no effect on the magnitude of changes in CK activity and muscle soreness. This may be of practical importance when designing training which includes drop jumps.

\section{References}

1. Brancaccio P., N.Maffulli, F.M.Limongelli (2007) Creatine kinase monitoring in sport medicine. Br.Med.Bull. 81:209-230.

2. Chen T.C. (2003) Effects of a second bout of maximal eccentric exercise on muscle damage and electromyographic activity. Eur.J.Appl.Physiol. 89:115-121.

3. Chen T.C., S.S.Hsieh (2001) Effects of a 7-day eccentric training period on muscle damage and inflammation. Med.Sci. Sports Exerc. 33:1732-1738.

4. Clarkson P.M., M.J.Hubal (2002) Exercise-induced muscle damage in humans. Am.J.Phys.Med.Rehabil. 81:S52-S69.

5. Ebben W.P., C.Simenz, R.L.Jensen (2008) Evaluation of plyometric intensity using electromyography. J.Strength Cond. Res. 22:861-868.

6. Hackney A.C., C.Battaglini (2007) The Overtraining Syndrome: Neuroendocrine imbalances in athletes. Braz.J.Biomot. 1:34-44.

7. Jensen R.L., W.P.Ebben (2007) Quantifying plyometric intensity via rate of force development, knee joint, and ground reaction forces. J.Strength Cond.Res. 21:763-767.

8. Mougios V. (2007) Reference intervals for serum creatine kinase in athletes. Br.J.Sports Med. 41:674-678.

9. Nosaka K., M.Newton, P.Sacco (2002) Muscle damage and soreness after endurance exercise of the elbow flexors. Med.Sci.Sports Exerc. 34:920-927.

10. Pereira R., M.Machado, M.Miragaya dos Santos, L.N.Pereira, F.Sampaio-Jorge, F. (2008) Muscle activation sequence compromises vertical jump performance. Serbian J.Sports Sci. 2:85-90.

11. Ribeiro V., R.Pereira, M.Machado (2008). Resistance exercise-induced microinjuries do not depend on 1 or 3 minutes rest time interval between series. Int.J.Sport Sci. 13:44-53.

12. Sankey S.P., P.A.Jones, T.M.Bampouras (2008) Effects of two plyometric training programs of different intensity on vertical jump performance in high school athletes. Serbian $J$. Sports Sci. 2:123-130.

13. Tidball J.G. (2008) Inflammation in skeletal muscle regeneration. In S.Schiaffino, T.Partridge (eds.) Skeletal Muscle Repair and Regeneration. Springer Science\& Business Media.

14. Twist C., R.G.Eston (2007) The effect of muscle-damaging exercise on maximal intensity cycling and drop jump performance. J.Exerc.Sci.Fitn. 5:1-9.

Received 9.07.2009

Accepted 24.10.2009

(c) University of Physical Education, Warsaw, Poland

Acknowledgements: Thanks are due to the participants and to Mr Edalmo França Jr. for blood sampling 